\title{
Fluorination-modulated end units for high- performance non-fullerene acceptors based organic solar cells
}

\author{
Yanna Sun ${ }^{\dagger}$, Huan-Huan Gao ${ }^{\dagger}$, Yuan-Qiu-Qiang Yi, Xiangjian Wan, Huanran Feng, Xin Ke, \\ Yamin Zhang, Jing Yan, Chenxi Li and Yongsheng Chen
}

Organic solar cells (OSCs) have drawn great attention for their low cost, light weight, solution processability and flexibility [1-3]. Over the last decade, fullerene and their derivatives have been the dominant electron acceptors for OSCs due to their excellent electron-transport properties, but limited by their disadvantages of poor light absorption, high synthesis cost, and difficulties in property tunability. To tackle these issues, design and synthesis of non-fullerene acceptors (NFAs) have received extensive attention and are urgently needed to explore for enhanced photovoltaic performance [4-9]. Among all the types of NFAs, acceptor-donor-acceptor (A-D-A) architecture based non-fullerene small molecule acceptors (NF-SMAs) shows tremendous potentials [10-13] and has achieved great advances for their excellent performance with power conversion efficiencies (PCEs) over $14 \%$ for single junction cells [14-18] and over $17 \%$ for tandem cells [19].

The giant success of A-D-A backboned NF-SMAs is mainly due to their well-defined chemical structures, easily tuned energy levels and facile synthetic procedures [20-32]. In previous studies, through delicately modulating the NF-SMAs, the high efficiency of non-fullerene organic photovoltaiscs (OPVs) has been obtained [3338]. As such, it is believed that there is a facile and effective way to finely tune properties of NFAs and further significantly enhance OPVs performance. The A-D-A backboned NF-SMAs mainly consisted of three segments: ladder-type electron-donating core, electron-withdrawing end units and outstretched side chains. Thus, modification of the above three components is an effective approach to obtain tunable energy levels, optical absorption, crystallinity, $\pi-\pi$ stacking and improved morphology of the active layers. In view of synthetic economy, the modification of electron-withdrawing end units is comparatively easy and facile as the attachment of the end units is typically the last step of the synthetic route [22,24,39-42].

Fluorination strategy has been widely applied in the organic semiconductor synthesis $[43,44]$. The roles of fluorine in OSCs materials have been extensively studied [45-48]. Firstly, fluorination enables strong electronwithdrawing ability due to strong electronegativity of fluorine, reduces the bandgap to near-infrared region and broadens the absorption range, which is one of the key determinants for achieving high short-circuit current density $\left(J_{\text {sc }}\right)[13,36]$. Secondly, fluorination can enhance $\pi-\pi$ stacking and crystallinity of the molecule to optimize morphology of the active layers and obtain appropriate domain size for achieving high mobility and fill factor (FF) $[22,29,40]$. Finally, fluorination would promote intermolecular interactions through forming noncovalent $\mathrm{F}-\mathrm{S}$ and $\mathrm{F}-\mathrm{H}$ bonds, which are favorable for efficient charge transport [43].

The A-D-A structured NF-SMAs based on fused benzo[1,2-b:4,5- $\left.b^{\prime}\right]$ dithiophene electron-donating core show large rigid and coplanar structure with strong electrondonating ability, both of which are beneficial to enhancing light absorption and charge transport $[28,33]$. In this work, three A-D-A structured NF-SMAs with end units modulated with different number of fluorine atoms and the benzo[1,2-b:4,5- $\left.b^{\prime}\right]$ dithiophene fused with diarylcyclopentadienylthieno[3,2- $b]$ thiophene (OBTT) as the core unit, namely, OBTT-0F, OBTT-2F and OBTT-4F

The Key Laboratory of Functional Polymer Materials, State Key Laboratory and Institute of Elemento-Organic Chemistry, Centre of Nanoscale Science and Technology, College of Chemistry, Nankai University, Tianjin 300071, China

${ }^{\dagger}$ These authors contributed equally to this work.

* Corresponding author (email: yschen99@nankai.edu.cn) 

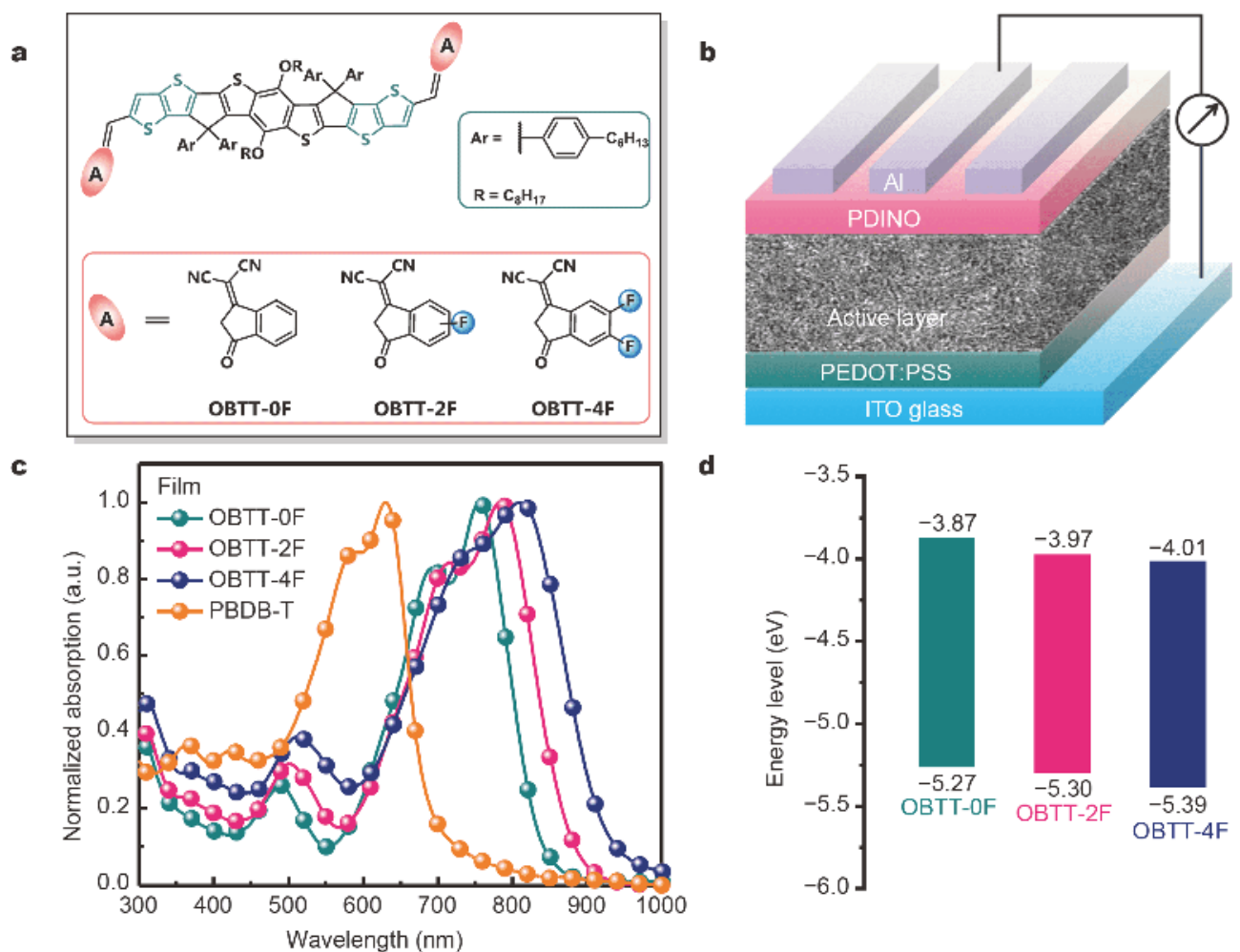

Figure 1 (a) Chemical structures of OBTT-0F, OBTT-2F and OBTT-4F. (b) Diagram of a conventional OSC. (c) Absorption spectra of OBTT-0F, OBTT-2F, OBTT-4F and PBDB-T in neat films. (d) Energy levels of the three acceptors.

(Fig. 1a), were designed and synthesized. We systematically characterized the optoelectronic properties and optimized the device performance of the three NF-SMAs. Fluorine modification on the end units leads to the significant downshift of the lowest unoccupied molecular orbital (LUMO) level and the absorption edge redshift of the NFAs. Moreover, the improvements of their crystallinity and enhanced $\pi-\pi$ stacking ameliorate morphology of the active layers, which contribute to a notably elevated $J_{\text {sc }}$ and FF of the corresponding OSCs. The OSCs based on OBTT-2F achieve a high PCE of $12.36 \%$ with a comparatively high $J_{\mathrm{sc}}$ of $20.83 \mathrm{~mA} \mathrm{~cm}{ }^{-2}$ and FF of $71.6 \%$. Our work reveals the vital role of the fluorination-modulated end units in A-D-A structured NF-SMAs on optoelectronic properties, charge transport, film morphology and photovoltaic properties, and provides valuable insights to optimize the structure and properties of the A-D-A structured NF-SMAs.

The desirable molecules OBTT-0F, OBTT-2F and OBTT-4F (Fig. 1a) were synthesized by Knoevenagel condensation between the dialdehyde intermediate and the corresponding end units via a five-step chemical reaction as displayed in Scheme S1, and the detailed syn- thetic procedures including characterization data are presented in the Supplementary information (SI). Though OBTT-2F is a mixture for the two isomers of end groups, consistent performance was observed between multiple batches. All three NF-SMAs are soluble in common organic solvents (such as dichloromethane, chloroform and chlorobenzene). As shown in Fig. S1, the decomposition temperatures of the three molecules at $5 \%$ weight loss are 325,332 and $328^{\circ} \mathrm{C}$ for OBTT-0F, OBTT-2F and OBTT$4 \mathrm{~F}$, respectively, indicating their good thermal stability.

The ultraviolet-visible-near-infrared (UV-vis-NIR) absorptions of these three NF-SMAs in their diluted solutions (Fig. S2) and thin films (Fig. 1c) were investigated (Table 1). As revealed in Fig. S2, in chloroform solutions, these three NFAs all exhibit strong and broad absorption in the region of $600-800 \mathrm{~nm}$ and the maximum absorption peaks of OBTT-0F, OBTT-2F and OBTT-4F are 746, 761 and $763 \mathrm{~nm}$, respectively. For the film (Fig. 1c), the absorption peaks of the NFAs with fluorine on the end units broaden with considerable redshift, due to the enhanced crystallinity and intermolecular $\pi-\pi$ stacking. It is worthwhile to note that there are strong shoulder peaks of the three NFAs, implying effective intermolecular $\pi-\pi$ 
Table 1 Basic properties of OBTT-0F, OBTT-2F and OBTT-4F

\begin{tabular}{|c|c|c|c|c|c|c|c|}
\hline Acceptors & $\lambda_{\max }^{\text {sol }}(\mathrm{nm})$ & $\lambda_{\max }^{\text {film }}(\mathrm{nm})$ & $\lambda_{\text {edge }}^{\text {film }}(\mathrm{nm})$ & $E_{\mathrm{g}}^{\text {opt }}(\mathrm{eV})^{\mathrm{a}}$ & HOMO (eV) & LUMO $(\mathrm{eV})$ & $E_{\mathrm{g}}^{\mathrm{CV}}(\mathrm{eV})^{\mathrm{b}}$ \\
\hline OBTT-0F & 746 & 756 & 856 & 1.45 & -5.27 & -3.87 & 1.40 \\
\hline OBTT-2F & 761 & 784 & 897 & 1.38 & -5.30 & -3.97 & 1.33 \\
\hline OBTT-4F & 763 & 811 & 945 & 1.31 & -5.39 & -4.01 & 1.38 \\
\hline
\end{tabular}

a) The optical band gap estimated from the absorption onset. b) Electrochemical bandgap obtained from $E_{\mathrm{LUMO}}-E_{\mathrm{HOMO}}$.

stacking. The intensive electron-withdrawing ability of fluorine makes a significant bathochromic-shifted spectrum for OBTT-0F, OBTT-2F and OBTT-4F. Thus, the optical bandgaps $\left(E_{\mathrm{g}}{ }^{\text {opt }}\right)$ of OBTT-0F, OBTT-2F and OBTT-4F, calculated from the absorption onset are 1.45, 1.38 , and $1.31 \mathrm{eV}$, respectively, and their absorption range is in near-infrared region, which is complementary to that of the wide bandgap polymer poly[(2,6-(4,8-bis(5-(2ethylhexyl)thiophen-2-yl)-benzo[1,2-b:4,5- $\left.b^{\prime}\right]$ dithiophene))-alt-(5,5-(1',3'-di-2-thienyl-5', $7^{\prime}$-bis(2-ethylhexyl) benzo[ $\left[1^{\prime}, 2^{\prime}-c: 4^{\prime}, 5^{\prime}-c^{\prime}\right]$ dithiophene-4,8-dione))] (PBDB-T) [47]. Their energy levels were estimated by electrochemical cyclic voltammetry (CV) referenced to the energy level of $\mathrm{Fc} / \mathrm{Fc}^{+}(-4.8 \mathrm{eV}$ below the vacuum level) (Fig. S3). The LUMO levels of OBTT-0F, OBTT-2F and OBTT-4F are $-3.87,-3.97$, and $-4.01 \mathrm{eV}$, respectively. These results indicate that fluorine modulation on the end units indeed downshifts the LUMO level, broadens the absorption range with redshifts to near-infrared region, in good agreement with the measured optical bandgaps.

To evaluate the potential photovoltaic performance of OBTT-0F, OBTT-2F and OBTT-4F as electron acceptors, solution-processed OSCs were fabricated with the conventional structure of indium tin oxide (ITO)/poly(3,4ethylenedioxythiopene):poly(styrenesulfonate) (PEDOT: PSS/PBDB-T:acceptors/perylene diimide functionalized with amino N-oxide (PDINO)/Al, where PDINO was selected as electron transport layer due to its suitable energy levels and electron extraction ability [49]. After systematic device optimizations (Tables S1-S3), chloroform was used as the solvent and 1,8-diiodooctane (DIO) was selected as solvent additive to tune the active layer morphology. The optimal amount of DIO is $0.5 \%, 0.3 \%$ and $0.3 \%$ volume, respectively, for the OBTT-0F, OBTT$2 \mathrm{~F}$ and OBTT-4F based OSCs. The total concentration of donor and acceptor was $11 \mathrm{mg} \mathrm{mL}^{-1}$ with donor:acceptor weight ratio of 1:1.2. The optimized device parameters are summarized in Table 2 with corresponding $J-V$ curves shown in Fig. 2a. The highest PCE of $12.36 \%$ is achieved by PBDB-T:OBTT-2F based devices along with a $V_{\text {oc }}$ of
$0.828 \mathrm{~V}, J_{\mathrm{sc}}$ of $20.83 \mathrm{~mA} \mathrm{~cm}^{-2}$, and $\mathrm{FF}$ of $71.6 \%$. DIO additive does not affect the $V_{\mathrm{oc}}$ notably but increases the $J_{\text {sc }}$ and FF for all OBTT-0F, OBTT-2F and OBTT-4F based OSCs, which results in higher PCEs. The performance of these three sets of OSCs clearly shows the effects of fluorination on the end units. The $V_{o c}$ is gradually reduced for the deeper LUMO level caused by electronwithdrawing effect of fluorine. However, due to smaller bandgap and broader absorption region, the absorption onset of OBTT-0F, OBTT-2F and OBTT-4F based OSCs extends from 850 to 900 and $950 \mathrm{~nm}$, which are accountable for the significantly increased $J_{\mathrm{sc}}$.

The external quantum efficiency (EQE) was measured to verify the higher $J_{s c}$ for OBTT-2F and OBTT-4F based devices. As shown in Fig. 2b, the integrated $J_{\text {sc }}$ of OBTT$0 \mathrm{~F}$, OBTT-2F and OBTT-4F based OSCs are in reasonable agreement with the measured $J_{\text {sc }}$ (Table 2). After fluorination-modulating the end units, as EQE is notably enhanced, OBTT-2F and OBTT- $4 \mathrm{~F}$ based OSCs obtain higher $J_{\text {sc }}$ than OBTT-0F based devices, which could be ascribed to their significantly redshifted absorption.

To investigate the effect of fluorination on electronic properties of these three NFAs, the electron and hole mobility of PBDB-T:OBTT-OF, PBDB-T:OBTT-2F and PBDB-T:OBTT-4F blend films were measured by the space-charge-limited current (SCLC) method using the electron-only and hole-only devices, respectively (Fig. S4). The calculated electron and hole mobility parameters for the corresponding blend films are $0.91 \times 10^{-4}, 1.29 \times 10^{-4}$ and $1.06 \times 10^{-4}\left(\mu_{\mathrm{e}}\right)$ and $1.28 \times 10^{-4}$, $1.47 \times 10^{-4}$ and $0.69 \times 10^{-4} \mathrm{~cm}^{-2} \mathrm{~V}^{-1} \mathrm{~s}^{-1}\left(\mu_{\mathrm{h}}\right)$, respectively. It should be noticed that PBDB-T:OBTT-2F based blend film has the highest electron mobility and most balanced carrier mobility among all films, contributing to the highest FF of $71.6 \%$ for PBDB-T:OBTT-2F based OSCs.

The exciton dissociation and charge collection behavior in the three OSCs were studied by measuring their photocurrent density $\left(J_{\mathrm{ph}}\right)$ as a function of the effective applied voltage $\left(V_{\text {eff }}\right)$ to tell the difference of $J_{\text {sc }}$ and FF among OBTT-0F, OBTT-2F, and OBTT-4F based OSCs. Herein, $J_{\mathrm{ph}}$ is calculated as $J_{\mathrm{ph}}=J_{\mathrm{L}}-J_{\mathrm{D}}$, where $J_{\mathrm{L}}$ and $J_{\mathrm{D}}$ are 

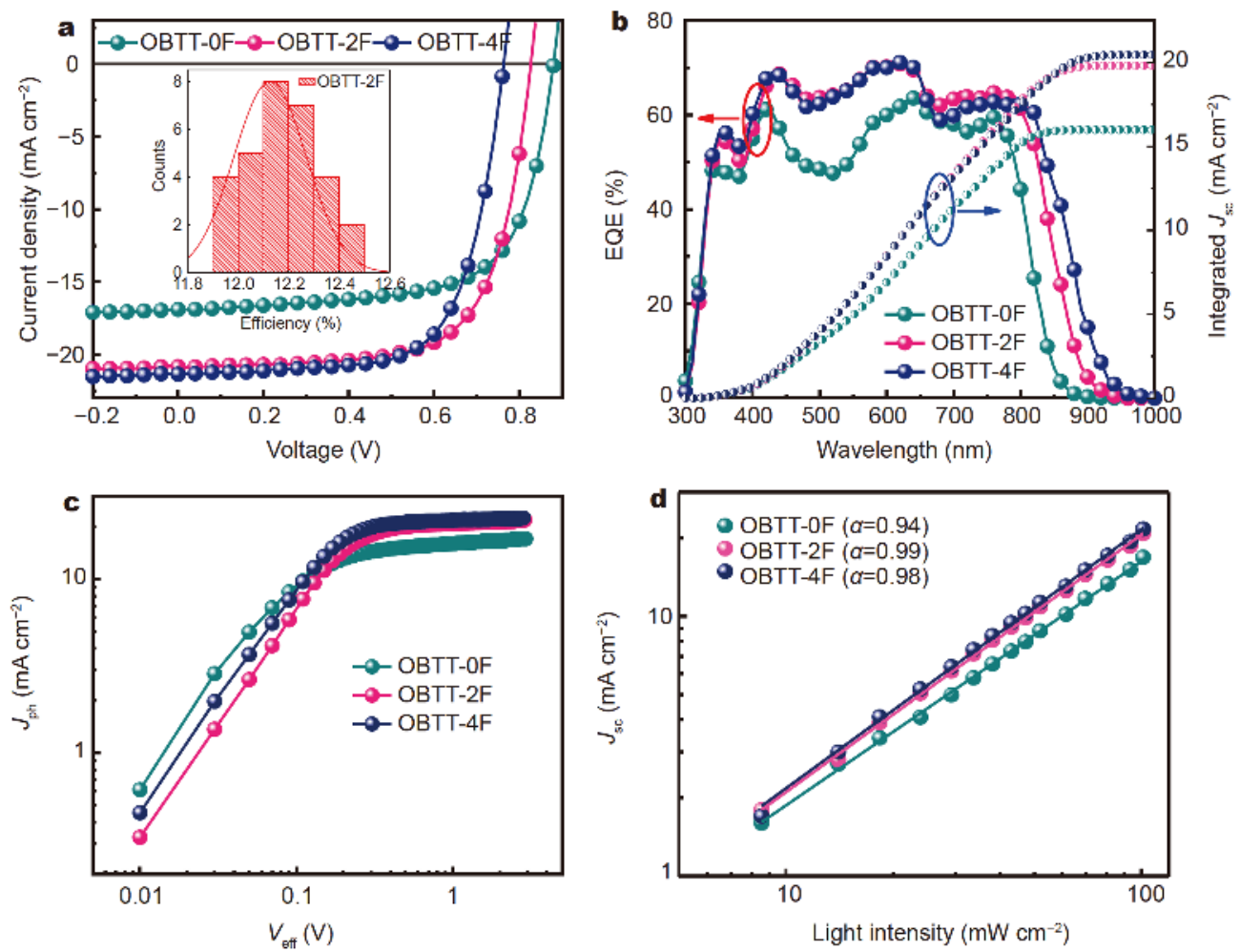

Figure 2 (a) Current density-voltage $(J-V)$ curves of the devices based on PBDB-T and OBTT-0F (2F, 4F) at optimized conditions under the illumination of AM 1.5G $\left(100 \mathrm{~mW} \mathrm{~cm}^{-2}\right)$; the inset shows the histograms of the PCE counts for 30 devices. (b) EQE spectra and integrated $J_{\mathrm{sc}}$ of the devices. (c) $J_{\mathrm{ph}}$ versus $V_{\text {eff }}$ and (d) light-intensity dependence of the devices' $J_{\text {sc. }}$.

Table 2 Device performance parameters of OSCs based on PBDB-T and three acceptors measured at simulated $100 \mathrm{~mW} \mathrm{~cm}^{-2} \mathrm{AM} 1.5 \mathrm{G}$ illumination

\begin{tabular}{|c|c|c|c|c|c|c|c|}
\hline \multirow{2}{*}{ Acceptors } & \multirow{2}{*}{ Treatment } & \multirow{2}{*}{$V_{\mathrm{oc}}(\mathrm{V})$} & \multicolumn{2}{|c|}{$J_{\mathrm{sc}}\left(\mathrm{mA} \mathrm{cm}^{-2}\right)$} & \multirow{2}{*}{ FF (\%) } & \multicolumn{2}{|c|}{ PCE (\%) } \\
\hline & & & Experimental & Calculated $^{\mathrm{a}}$ & & Best & Average $^{\mathrm{b}}$ \\
\hline \multirow[t]{2}{*}{ OBTT-0F } & As-cast & 0.902 & 13.92 & & 58.2 & 7.31 & 7.30 \\
\hline & $0.50 \% \mathrm{DIO}$ & 0.889 & 16.86 & 16.02 & 67.9 & 10.20 & 10.16 \\
\hline \multirow[t]{2}{*}{ OBTT-2F } & As-cast & 0.827 & 18.32 & & 61.1 & 9.26 & 9.17 \\
\hline & $0.30 \% \mathrm{DIO}$ & 0.828 & 20.83 & 19.9 & 71.6 & 12.36 & 12.10 \\
\hline \multirow[t]{2}{*}{ OBTT-4F } & As-cast & 0.777 & 18.96 & & 57.5 & 8.48 & 8.42 \\
\hline & $0.30 \%$ DIO & 0.772 & 21.67 & 20.5 & 69.1 & 11.55 & 11.43 \\
\hline
\end{tabular}

a) The values are integrated $J_{\text {sc }}$ from EQE spectra. b) Average values from 30 devices.

the current densities under light and in the dark, while $V_{\text {eff }}$ is defined as $V_{\text {eff }}=V_{0}-V_{\text {app }}$, where $V_{0}$ is the voltage when $J_{\mathrm{ph}}=0$ and $V_{\text {app }}$ is the applied external voltage. As depicted in Fig. 2c, when $V_{\text {eff }}$ exceeded $1.5 \mathrm{~V}, J_{\text {ph }}$ of the three OSCs reached saturation $\left(J_{\text {sat }}\right)$, indicating that the charge recombination is minimized at high voltages due to the high internal electric field in the devices. To further compare the charge dissociation and charge collection probability $(P(E, T))$ in the three devices, where $E$ and $T$ represent field and temperature respectively, the value of $\left(\mathrm{J}_{\mathrm{ph}} / \mathrm{J}_{\mathrm{sat}}\right)$ was adopted. Under the short-circuit conditions, compared with $92 \%$ for the OBTT-0F based devices, the $J_{\text {ph }} / J_{\text {sat }}$ ratios were $96 \%$ and $95 \%$ for the OBTT-2F and OBTT-4F-based devices, suggesting introducing fluorine to the end units could lead to high exciton dissociation and charge collection efficiencies and thus the high FFs.

Furthermore, we investigated the charge recombination property in the OBTT-OF, OBTT-2F, and OBTT-4F based 
OSCs by measuring the relationship between the $J_{\mathrm{sc}}$ and the light intensity $(P)$. The relationship between $J_{\mathrm{sc}}$ and $P$ can be described with the formula of $J_{\mathrm{sc}} \propto P^{\alpha}$, where power law exponent $\alpha$ implies the extent of bimolecular recombination. Weak bimolecular recombination in the device would result in a linear dependence of $J_{\text {sc }}$ on light intensity with an $\alpha$ of $\sim 1$. As shown in Fig. 2d, the $\alpha$ for the devices based on PBDB-T:OBTT-0F, OBTT-2F and OBTT-4F are 0.94, 0.99, and 0.98, respectively. All the values of $\alpha$ are very close to 1, and OBTT-2F and OBTT4 F-based devices demonstrate higher $\alpha$, implying that through introduction of fluorine atoms on the end units, less bimolecular recombination occurred in the optimized devices.

The morphology of films based on OBTT-0F, OBTT-2F and OBTT-4F was investigated by grazing incidence wide-angle X-ray scattering (GIWAXS). The 2D GIWAXS patterns and cut-line profiles of the in-plane and out-of-plane directions are shown in Fig. 3. In the OBTT$0 F$ neat film, weak (100) lamellar diffraction peak appears at $\sim 0.32 \AA^{-1}$ with $\pi-\pi$ stacking diffraction peak $(010)$ at around $1.79 \AA^{-1}$, indicating that it is highly amorphous in the pure film. After introducing fluorine to the end units, OBTT-2F and OBTT-4F pure films exhibit strong $\pi-\pi$ stacking (010) diffraction peaks at 1.80 and $1.81 \AA^{-1}$, respectively. These results reflect that fluorination-modulated end units enhance the $\pi-\pi$ stacking of A-D-A structured NFAs. As for PBDB-T:NFAs blend films (Fig. 3d-f), the PBDB-T:OBTT-0F blend film shows strong (010) diffraction peaks in $q_{z}$ direction of the polymer donor but weak peak of the OBTT-0F [50]. However, in the OBTT-2F and OBTT-4F based blend films, they show dominant face-on orientations with intense (010) diffraction peaks at 1.73 and $1.74 \AA^{-1}$, respectively, with the crystal coherence length (CCL) of 62.8 and $37.7 \AA^{-1}$ by Gaussian fitting. The GIWAXS result reveals that fluorination-modulated end units can enhance $\pi-\pi$ stacking and crystallinity of the NF-SMAs, which is beneficial to high charge mobility and FF.

To further investigate the phase separation morphologies of the three photoactive layers, atomic force microscopy (AFM) and transmission electron microscopy (TEM) were carried out. As shown in Fig. 4a, d, g, the PBDB-T:OBTT-0F based active layer shows smooth morphology with small root-mean-square (RMS) roughness $(0.83 \mathrm{~nm}$, Fig. $4 \mathrm{a})$. No large phase separation was observed from its TEM image. The PBDB-T:OBTT-2F and PBDB-T:OBTT-4F films gave larger $R_{\mathrm{q}}$ values of 1.76 and $2.82 \mathrm{~nm}$ (Fig. $4 \mathrm{~b}, \mathrm{c}$ ), indicating more ordered nanoscale morphology in the blend films. Meanwhile, the AFM phase and TEM images of the PBDB-T:OBTT-2F (Fig. 4e, h) and PBDB-T:OBTT-4F (Fig. 4f, i) films show larger and more continuous grain-like domains with proper size, which is more favorable for charge transport and collection.

In summary, to investigate the effect of fluorinationmodulated end units, three A-D-A structured NF-SMAs of OBTT-0F, OBTT-2F and OBTT-4F are designed and synthesized based on end units modulated with $0,2,4$ fluorine atom(s). OBTT-0F, OBTT-2F and OBTT-4F all
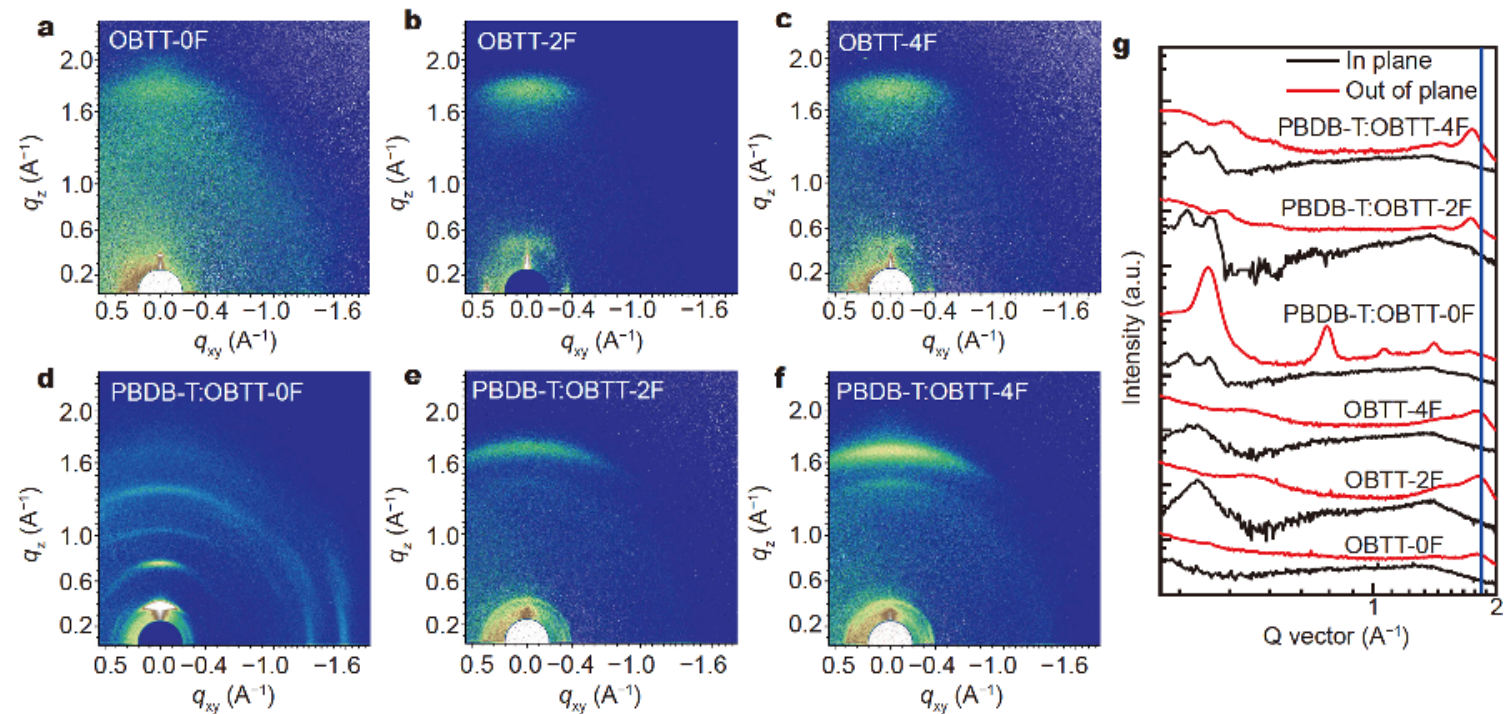

Figure 3 2D-GIWAXS patterns for (a) OBTT-OF, (b) OBTT-2F and (c) OBTT-4F neat film, (d) PBDB-T:OBTT-0F, (e) PBDB-T:OBTT-2F and (f) PBDB-T:OBTT-4F blend film. (g) Out-of-plane and in-plane GIWAXS profiles for the neat and blend films. 


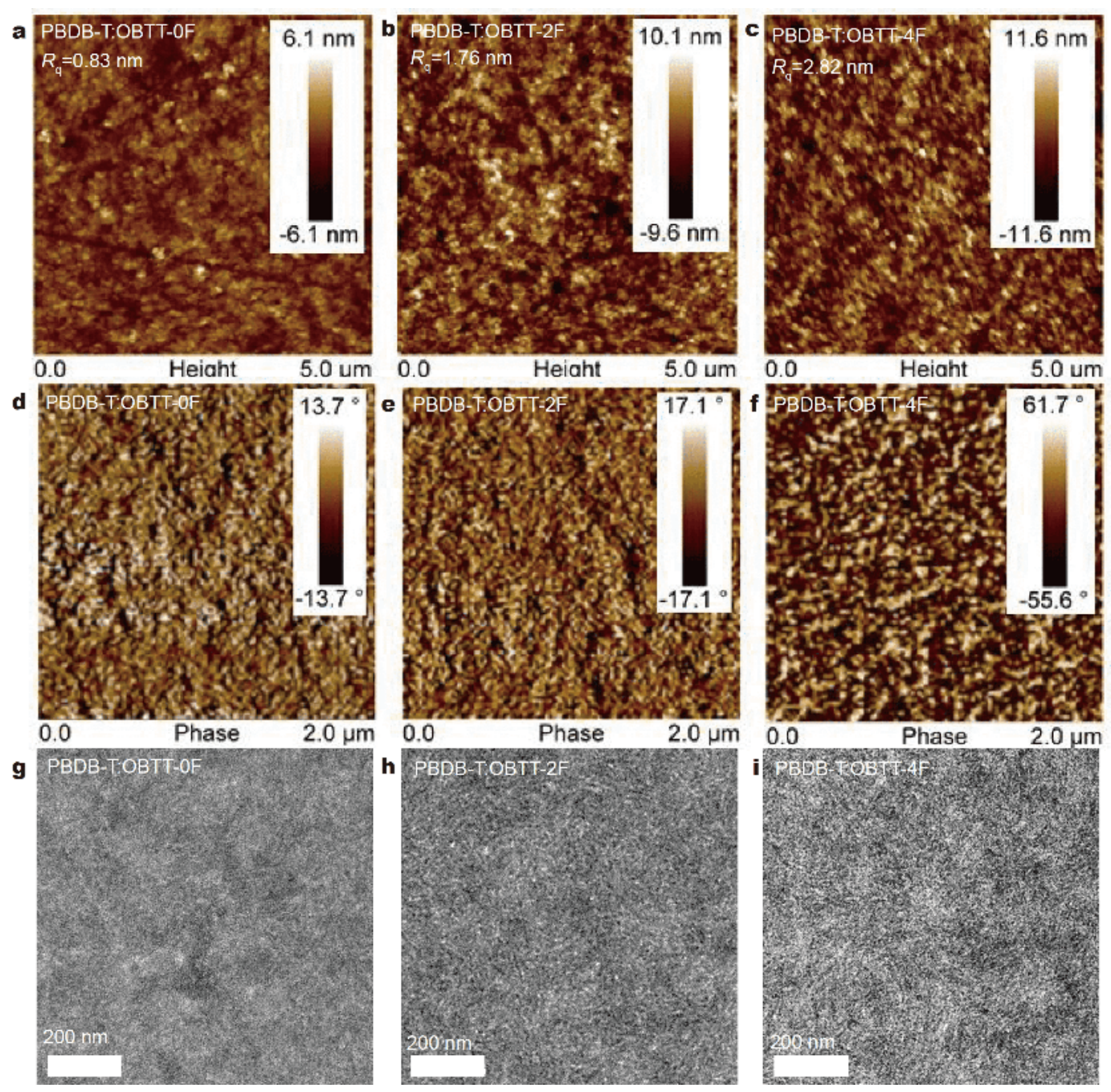

Figure 4 Tapping-mode AFM height and phase images and TEM images of (a, d, g) PBDB-T:OBTT-OF, (b, e, h) PBDB-T:OBTT-2F and (c, f, i) PBDB-T:OBTT-4F blend films.

have broad absorption in near-infrared region and show low bandgaps of $1.45,1.38$, and $1.31 \mathrm{eV}$, respectively. Through introduction of fluorine on the end units, the absorption edge of the NFAs can significantly redshift with the $\pi-\pi$ stacking and crystallinity of the molecule notably enhanced, although it also simultaneously causes some downshift of energy levels of NFAs. Therefore, fluorination on the end units results in lower $V_{\text {oc }}$ but significantly higher $J_{\mathrm{sc}}$ and FF of the corresponding devices. Overall, the two contrary factors endow the OBTT$2 \mathrm{~F}$ based OSCs with a high PCE of $12.36 \%$ notably better than the OSCs based on OBTT-0F and OBTT-4F. Our work indicates that delicate and fine tuning of molecular structure could realize high performance of OPV.
Received 4 February 2019; accepted 14 March 2019; published online 10 April 2019

1 Yu G, Gao J, Hummelen JC, et al. Polymer photovoltaic cells: Enhanced efficiencies via a network of internal donor-acceptor heterojunctions. Science, 1995, 270: 1789-1791

2 Li G, Zhu R, Yang Y. Polymer solar cells. Nat Photon, 2012, 6: 153-161

3 Lin Y, Zhan X. Oligomer molecules for efficient organic photovoltaics. Acc Chem Res, 2016, 49: 175-183

4 Hou J, Inganäs $\mathrm{O}$, Friend $\mathrm{RH}$, et al. Organic solar cells based on non-fullerene acceptors. Nat Mater, 2018, 17: 119-128

5 Cheng P, Li G, Zhan X, et al. Next-generation organic photovoltaics based on non-fullerene acceptors. Nat Photon, 2018, 12: 131-142

6 Zhang G, Zhao J, Chow PCY, et al. Nonfullerene acceptor molecules for bulk heterojunction organic solar cells. Chem Rev, 2018, 118: $3447-3507$ 
7 Nielsen CB, Holliday S, Chen HY, et al. Non-fullerene electron acceptors for use in organic solar cells. Acc Chem Res, 2015, 48: 2803-2812

8 Chen Y, Wan X, Long G. High performance photovoltaic applications using solution-processed small molecules. Acc Chem Res, 2013, 46: 2645-2655

9 Yang Y, Wang K, Li G, et al. Fluorination triggered new small molecule donor materials for efficient as-cast organic solar cells. Small, 2018, 14: 1801542

10 Xiao B, Tang A, Yang J, et al. Quinoxaline-containing nonfullerene small-molecule acceptors with a linear $A_{2}-A_{1}-D-A_{1}-A_{2}$ skeleton for poly(3-hexylthiophene)-based organic solar cells. ACS Appl Mater Interfaces, 2018, 10: 10254-10261

11 Tang A, Xiao B, Wang Y, et al. Simultaneously achieved high open-circuit voltage and efficient charge generation by fine-tuning charge-transfer driving force in nonfullerene polymer solar cells. Adv Funct Mater, 2018, 28: 1704507

12 Li J, Yang J, Hu J, et al. The first thieno[3,4-b]pyrazine based small molecular acceptor with a linear $\mathrm{A}_{2}-\mathrm{A}_{1}-\mathrm{D}-\mathrm{A}_{1}-\mathrm{A}_{2}$ skeleton for fullerene-free organic solar cells with a high $V_{\text {oc }}$ of $1.05 \mathrm{~V}$. Chem Commun, 2018, 54: 10770-10773

13 Tang A, Xiao B, Chen F, et al. The introduction of fluorine and sulfur atoms into benzotriazole-based p-type polymers to match with a benzotriazole-containing n-type small molecule: "The sameacceptor-strategy" to realize high open-circuit voltage. Adv Energy Mater, 2018, 8: 1801582

14 Zhang Y, Yao H, Zhang S, et al. Fluorination vs. chlorination: A case study on high performance organic photovoltaic materials. Sci China Chem, 2018, 61: 1328-1337

15 Zhang S, Qin Y, Zhu J, et al. Over 14\% efficiency in polymer solar cells enabled by a chlorinated polymer donor. Adv Mater, 2018, 30: 1800868

16 Zhang H, Yao H, Hou J, et al. Over $14 \%$ efficiency in organic solar cells enabled by chlorinated nonfullerene small-molecule acceptors. Adv Mater, 2018, 30: 1800613

17 Kan B, Feng H, Yao H, et al. A chlorinated low-bandgap smallmolecule acceptor for organic solar cells with $14.1 \%$ efficiency and low energy loss. Sci China Chem, 2018, 61: 1307-1313

18 Yuan J, Zhang Y, Zhou L, et al. Single-junction organic solar cell with over 15\% efficiency using fused-ring acceptor with electrondeficient core. Joule, 2019, doi: 10.1016/j.joule.2019.01.004

19 Meng L, Zhang Y, Wan X, et al. Organic and solution-processed tandem solar cells with 17.3\% efficiency. Science, 2018, 361: 10941098

20 Zhu J, Ke Z, Zhang Q, et al. Naphthodithiophene-based nonfullerene acceptor for high-performance organic photovoltaics: Effect of extended conjugation. Adv Mater, 2018, 30: 1704713

21 Gao W, Zhang M, Liu T, et al. Asymmetrical ladder-type donorinduced polar small molecule acceptor to promote fill factors approaching $77 \%$ for high-performance nonfullerene polymer solar cells. Adv Mater, 2018, 30: 1800052

22 Zhao F, Dai S, Wu Y, et al. Single-junction binary-blend nonfullerene polymer solar cells with $12.1 \%$ efficiency. Adv Mater, 2017, 29: 1700144

23 Yi YQQ, Feng H, Chang M, et al. New small-molecule acceptors based on hexacyclic naphthalene(cyclopentadithiophene) for efficient non-fullerene organic solar cells. J Mater Chem A, 2017, 5: 17204-17210

24 Yao H, Ye L, Hou J, et al. Achieving highly efficient nonfullerene organic solar cells with improved intermolecular interaction and open-circuit voltage. Adv Mater, 2017, 29: 1700254

25 Xu SJ, Zhou Z, Liu W, et al. A twisted thieno[3,4-b]thiophenebased electron acceptor featuring a 14- $\pi$-electron indenoindene core for high-performance organic photovoltaics. Adv Mater, 2017, 29: 1704510

26 Ma Y, Zhang M, Yan Y, et al. Ladder-type dithienonaphthalenebased small-molecule acceptors for efficient nonfullerene organic solar cells. Chem Mater, 2017, 29: 7942-7952

27 Li Y, Zhong L, Gautam B, et al. A near-infrared non-fullerene electron acceptor for high performance polymer solar cells. Energy Environ Sci, 2017, 10: 1610-1620

28 Kan B, Feng H, Wan X, et al. Small-molecule acceptor based on the heptacyclic benzodi(cyclopentadithiophene) unit for highly efficient nonfullerene organic solar cells. J Am Chem Soc, 2017, 139: 4929-4934

29 Dai S, Zhao F, Zhang Q, et al. Fused nonacyclic electron acceptors for efficient polymer solar cells. J Am Chem Soc, 2017, 139: 13361343

30 Zhao W, Qian D, Zhang S, et al. Fullerene-free polymer solar cells with over $11 \%$ efficiency and excellent thermal stability. Adv Mater, 2016, 28: 4734-4739

31 Lin Y, Li T, Zhao F, et al. Structure evolution of oligomer fusedring electron acceptors toward high efficiency of as-cast polymer solar cells. Adv Energy Mater, 2016, 6: 1600854

32 Lin Y, Wang J, Zhang ZG, et al. An electron acceptor challenging fullerenes for efficient polymer solar cells. Adv Mater, 2015, 27: $1170-1174$

33 Wang J, Zhang J, Xiao Y, et al. Effect of isomerization on highperformance nonfullerene electron acceptors. J Am Chem Soc, 2018, 140: 9140-9147

34 Zhao W, Ye L, Li S, et al. Environmentally-friendly solvent processed fullerene-free organic solar cells enabled by screening halogen-free solvent additives. Sci China Mater, 2017, 60: 697-706

35 Gao HH, Sun Y, Wan X, et al. Design and synthesis of low band gap non-fullerene acceptors for organic solar cells with impressively high $J_{\mathrm{sc}}$ over $21 \mathrm{~mA} \mathrm{~cm}{ }^{-2}$. Sci China Mater, 2017, 60: 819-828

36 Shi X, Chen J, Gao K, et al. Terthieno[3,2-b]thiophene (6T) based low bandgap fused-ring electron acceptor for highly efficient solar cells with a high short-circuit current density and low open-circuit voltage loss. Adv Energy Mater, 2018, 8: 1702831

37 Chen Y, Liu T, Hu H, et al. Modulation of end groups for lowbandgap nonfullerene acceptors enabling high-performance organic solar cells. Adv Energy Mater, 2018, 8: 1801203

38 Feng H, Qiu N, Wang X, et al. An A-D-A type small-molecule electron acceptor with end-extended conjugation for high performance organic solar cells. Chem Mater, 2017, 29: 7908-7917

39 Luo Z, Bin H, Liu T, et al. Fine-tuning of molecular packing and energy level through methyl substitution enabling excellent small molecule acceptors for nonfullerene polymer solar cells with efficiency up to $12.54 \%$. Adv Mater, 2018, 30: 1706124

40 Zhao W, Li S, Yao H, et al. Molecular optimization enables over $13 \%$ efficiency in organic solar cells. J Am Chem Soc, 2017, 139: 7148-7151

41 Li S, Ye L, Zhao W, et al. Significant influence of the methoxyl substitution position on optoelectronic properties and molecular packing of small-molecule electron acceptors for photovoltaic cells. Adv Energy Mater, 2017, 7: 1700183

42 Li S, Ye L, Zhao W, et al. Design of a new small-molecule electron acceptor enables efficient polymer solar cells with high fill factor. 
Adv Mater, 2017, 29: 1704051

43 Babudri F, Farinola GM, Naso F, et al. Fluorinated organic materials for electronic and optoelectronic applications: The role of the fluorine atom. Chem Commun, 2007, 105: 1003-1022

44 Tang ML, Bao Z. Halogenated materials as organic semiconductors. Chem Mater, 2011, 23: 446-455

45 Sakamoto Y, Komatsu S, Suzuki T. Tetradecafluorosexithiophene: The first perfluorinated oligothiophene. J Am Chem Soc, 2001, 123: 4643-4644

46 Lei T, Xia X, Wang JY, et al. "Conformation locked" strong electron-deficient poly ( $p$-phenylene vinylene) derivatives for ambientstable n-type field-effect transistors: Synthesis, properties, and effects of fluorine substitution position. J Am Chem Soc, 2014, 136: 2135-2141

47 Kim HG, Kang B, Ko H, et al. Synthetic tailoring of solid-state order in diketopyrrolopyrrole-based copolymers via intramolecular noncovalent interactions. Chem Mater, 2015, 27: 829-838

48 Sun SX, Huo Y, Li MM, et al. Understanding the halogenation effects in diketopyrrolopyrrole-based small molecule photovoltaics. ACS Appl Mater Interfaces, 2015, 7: 19914-19922

49 Zhang ZG, Qi B, Jin Z, et al. Perylene diimides: A thicknessinsensitive cathode interlayer for high performance polymer solar cells. Energy Environ Sci, 2014, 7: 1966

50 Yi YQQ, Feng H, Zheng N, et al. Small molecule acceptors with a nonfused architecture for high-performance organic photovoltaics. Chem Mater, 2019, 31: 904-911

Acknowledgements This work was supported by the Ministry of Science and Technology (2016YFA0200200), the National Natural Science Foundation of China (91633301 and 51773095), the Natural Science Foundation of Tianjin City (17JCJQJC44500 and 17CZDJC31100) and 111 Project (B12015). This work is dedicated to the $100^{\text {th }}$ anniversary of Nankai University.

Author contributions Sun $\mathrm{Y}$, Chen $\mathrm{Y}$ and Gao HH designed the project; Wan X, Li C and Chen Y directed the research; Sun Y, Yi YQ, Feng H, Zhang Y and Yan J fabricated and characterized the devices; Ke $\mathrm{X}$ finished the TGA characterization. Sun $\mathrm{Y}$ wrote the paper, with support from Chen Y. All authors contributed to the general discussion.

Conflict of interest The authors declare no conflict of interest.

Supplementary information Experimental details and supporting data are available in the online version of this paper.

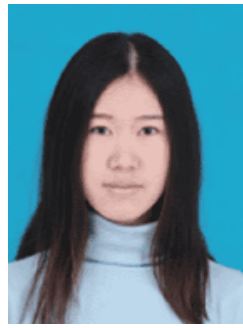

Yanna Sun is a $\mathrm{PhD}$ candidate under the supervision of Prof. Yongsheng Chen at Nankai University. She received her bachelor's degree in polymeric materials science from Ocean University of China in 2015. Her research focuses on the design and synthesis of organic photovoltaic materials.

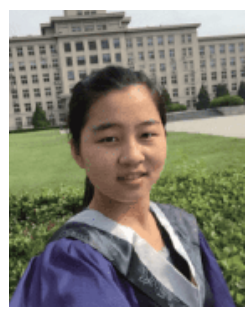

Huan-Huan Gao is a $\mathrm{PhD}$ candidate under the supervision of Prof. Yongsheng Chen and Xiangjian Wan at Nankai University. She received her bachelor's degree in chemistry from Nanyang Normal University in 2013 and master's degree in organic chemistry from Nankai University in 2016. Her research focuses on the design and synthesis of organic photovoltaic materials.

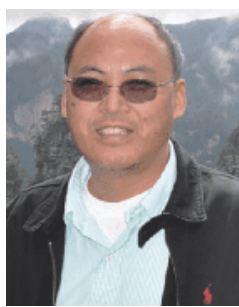

Yongsheng Chen received his $\mathrm{PhD}$ in chemistry at the University of Victoria in 1997. From 2003, he has been a Chair Professor at Nankai University. His main research interests focus on the carbon-based nanomaterials and organic functional materials for green energy applications.

\section{氟修饰端基非富勒烯受体高效率有机太阳能电池}

孙延娜 ${ }^{\dagger}$, 高欢欢 ${ }^{\dagger}$, 易袁秋强, 万相见, 冯焕然, 柯金金, 张雅敏, 燕晶, 李晨曦, 陈永胜 ${ }^{*}$

摘要 本文设计合成了三个受体-给体-受体骨架的非富勒烯受体, 它们具有不同数目氟原子修饰的端基, 将其分别命名为 OBTT-0F, OBTT-2F和OBTT-4F. 氟端基使得最低未占分子轨道下移, 光谱红 移, $\pi-\pi$ 堆叠和非富勒烯受体的结晶性增强. 通过与聚合物给体 PBDB- $\mathrm{T}$ 共混, 基于 $\mathrm{OBTT}-2 \mathrm{~F}$ 的分子同时获得了相对高的电流 $\left(20.83 \mathrm{~mA} \mathrm{~cm}^{-2}\right.$ ) 和能量转换效率(12.36\%). 该结果证明向端基上引 入氟原子是调控非富勒烯受体光电性能和光伏效率的简单有效的 方法. 